\title{
URGENCY TREATMENT PROFILE OF 0 TO 15 YEAR-OLD CHILDREN ASSISTED AT URGENCY DENTAL SERVICE FROM BAURU DENTAL SCHOOL, UNIVERSITY OF SÃO PAULO
}

\author{
PERFIL DE TRATAMENTO DE URGENCIA DE CRIANÇAS DE 0 A 15 ANOS ATENDIDAS NO \\ SERVIÇO DE URGÊNCIA ODONTOLÓGICA DA FACULDADE DE ODONTOLOGIA DE \\ BAURU, UNIVERSIDADE DE SÃO PAULO
}

Vivien Thiemy SAKAI ${ }^{1}$, Ana Carolina MAGALHÃES ${ }^{1}$, Juliano Pelim PESSAN ${ }^{1}$, Salete Moura Bonifácio da SILVA², Maria Aparecida de Andrade Moreira MACHADO²

1- DDS, MSc, Graduate student (Master degree), Department of Pediatric Dentistry, Orthodontics and Public Health, Bauru Dental School, University of São Paulo.

2- DDS, PhD, Associate Professor, Department of Pediatric Dentistry, Orthodontics and Public Health, Bauru Dental School, University of São Paulo.

Corresponding Address: Maria Aparecida de Andrade Moreira Machado - Al. Octávio Pinheiro Brisolla, 9-75 - Vl. Universitária - Bauru - SP Brazil - CEP 17012-901 - Phone \#: 551432358218 Fax \#: 551432234679 - E-mail: mmachado@fob.usp.br

Received: October 26, 2004 - Modification: April 04, 2005 - Accepted: April 08, 2005

\begin{abstract}
D ntal recordings of 0 to 15-year-old patients assisted at Urgency Dental Service (UDS) from Bauru Dental School, University of São Paulo, in 2001 and 2002, were assessed in order to quantify the number of patients that used the service, to determine attendance patterns, and to record the frequency of different types of dental emergencies and their performed treatment. Data were plotted and submitted to a descriptive statistical analysis. Among the total of patients attended at UDS (6020), 1166 (19.37\%) were children, with mean age of 9.24 years. Trauma was the cause for 199 (17.06\%) of the recorded urgency visits. It occurred more frequently in children between 0 and 3 years of age (34.42\%), and between 7 and 12 years of age (18.12\%). The main treatments performed were temporary restoration (33.33\%) for coronal fracture, and orientation (24.44\%) for luxation. Nontraumatic events were the etiology for 967 (82.92\%) of the total urgency diagnosis. The most commonly found nontraumatic diagnosis was dental caries lesions (61.75\%), followed by problems of eruption or root resorption (14.27\%) and bone or soft tissue lesions (6.51\%), among others (17.47\%). The most frequent treatments performed for caries lesions were: excavation and temporary restoration (39.39\%) when there was no abscess, and coronal opening and dressing (40.95\%) for caries lesions with abscess. There was an increasing trend in caries lesions prevalence according to the rising of the age, in contrast to trauma prevalence. Treatment for both situations was done according to the indicated protocol for each case.

Uniterms: Emergency treatment; Dental care for children; Tooth injuries; Dentistry.
\end{abstract}

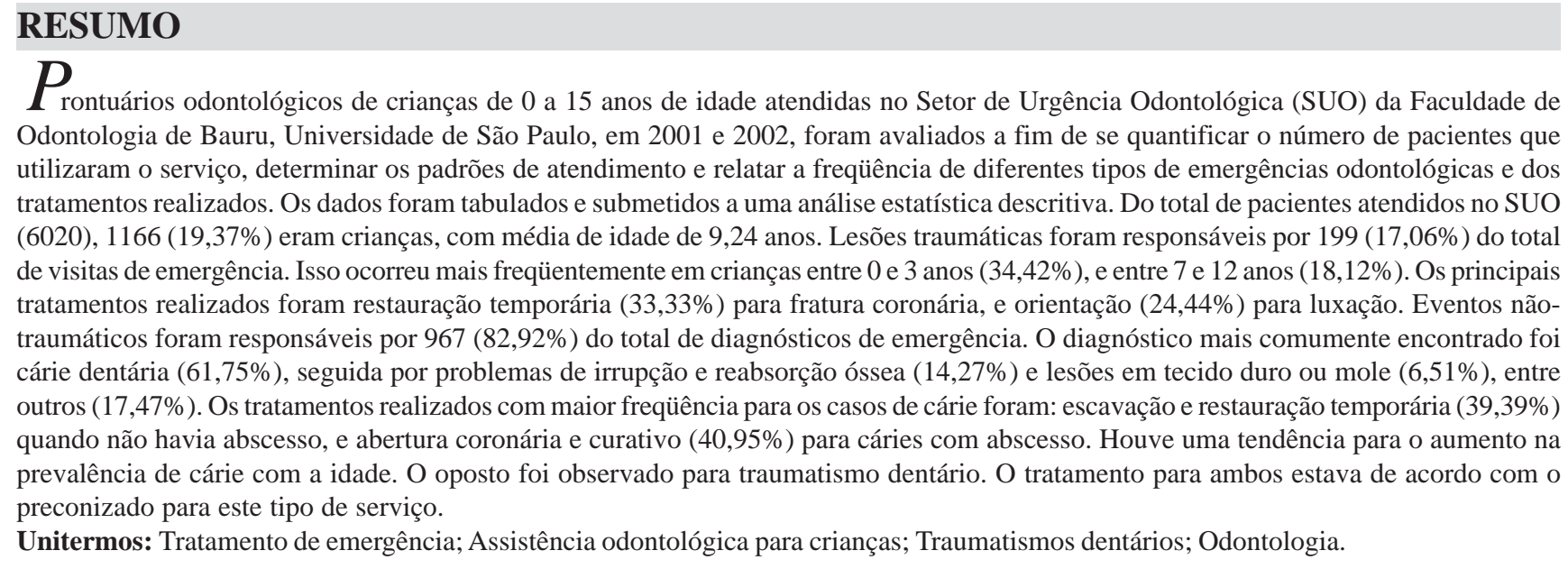




\section{INTRODUCTION}

Although Dentistry has emphasized the prevention of oral diseases in the last years, most of them are still common in our population, indeed there are a number of barriers for seeking of oral health care in Brazil. Due to the lack of information and resources, people just seek treatment when the problem becomes really serious, and they are feeling some discomfort or pain. In order to solve their problem immediately, people use dental urgency service as a primary care source.

The urgency dental service can be defined as the care of patients that present oral problems which interfere in their lives or in the organs functioning, such as jaw and alveolar bone fractures, avulsed or displaced teeth, fractured teeth with pulp exposures, acute alveolar abscess, upper airway impairment, oral mucosal lacerations, acute dental pain and infection, and uncontrolled bleeding ${ }^{2,13}$.

Some of the patients that seek this type of attendance are children. Schwartz ${ }^{15}$, in 1994, stated that there is a great variety of causes for urgency dental visits among the pediatric population, and a classification system has been developed in a comprehensive and orderly manner. Agostini, Flaitz and Hicks $^{2}$, in 2001, in a study with 816 children, whose ages ranged from 10 days to 15 years, confirmed that most children seeking urgency dental services (65.5\%) were six years of age or younger.

Although dental emergencies are a common occurrence in pediatric dentistry practices ${ }^{2,3,15}$, data from children profile that search this kind of attendance are rare, and generally, when they are performed, they happen in hospitals ${ }^{16}$. Despite its importance, little is known regarding the prevalence and etiology of urgency dental visits in non-hospital settings ${ }^{2}$. Moreover, little information is available about the treatment provided to these patients in urgency situations ${ }^{17}$, and sometimes most patients are treated empirically according to their presenting signs and symptoms ${ }^{11}$.

The purpose of the present study was to assess the urgency treatment profile of 0 to 15 year-old children, attended at Urgency Dental Service from Bauru School of Dentistry, University of São Paulo (UDS), a 2-year period study determining the prevalence and necessities of this population, as well as the treatment performed.

\section{MATERIALAND METHODS}

The urgency records of 0 to 15 year-old children, who presented to UDS from Bauru Dental School, University of
São Paulo with oral problems, during the years of 2001 and 2002, were reviewed. The following data were collected: age and gender, main complaint and diagnosis, symptoms, duration of the problem, exams and treatments performed, and followup. Just the first attendance of patients were considered, except when subsequent attendances were necessary to solve the problems with which they attended for care.

To record the etiology of the urgency attendance, the problems that prompted each patient's visit were divided into traumatic and nontraumatic causes ${ }^{2,5}$. The most implicated teeth in these causes were recorded. In case of trauma of multiple areas, the area of the most severe injury was recorded ${ }^{2}$. Traumarelated diagnostic categories included luxation, intrusion, extrusion, avulsion, coronal fracture, radicular fracture, bone fracture and resorption and soft tissue lesion. Nontrauma diagnostic categories included dental caries lesions without abscess formation, dental caries lesions with abscess formation, eruption-related complications, bone and soft tissue lesions, and others.

The symptoms that could be associated to all these problems were pain, swelling, bleeding, infection and others. This item could be fulfilled by more than one symptom. Beside the causal factors and symptoms, the duration of the problem was another item that was assessed, since it could interfere with the treatment. In this study, the duration was divided in the following categories: less than 1 day, 1 to 2 days, 3 to 7 days, more than 7 days.

To determine the most prevalent age groups and to examine the relationship between age and nature of the urgency (trauma vs nontrauma), four age categories were established: G1 - 0 to 3 years, G2 -4 to 6 years; G3 -7 to 12 years; and G4 -13 to 15 years of age ${ }^{5}$.

Regarding the treatment performed, information obtained from the clinical records of UDS were considered and organized according to the categories previously determined for this study according to the type of the injury (Table 1).

It was also recorded if the patient was referred to another clinic from Bauru Dental School to provide a regular dental care and/or to follow up the urgency treatment performed.

Data were submitted to a descriptive statistical analysis.

\section{RESULTS}

The population of this study (1166 children), with average age of 9.24 years, represented $19.37 \%$ of all patients attended at UDS (6020) during the 24-month interval. There was no gender predilection: 586 were males (50.25\%) and 580 were

TABLE 1- Relationship between causes of urgency attendance and possible treatments to be performed

Caries lesions without abscess

Caries lesions with abscess

Trauma

Other
Excavation, coronal opening and dressing, extraction, no treatment Medication (antibiotic), coronal opening and dressing, extraction, no treatment Follow-up, retention, coronal opening and dressing, extraction, no treatment Orientation, follow-up, medication, surgery, another treatment 
females (49.74\%).

Most children seeking dental urgency treatment at UDS were from G3 (7 to 12 years old -42.97\%), followed by G4 (13 to 15 years old $-27.28 \%$ ), G2 (4 to 6 years old $-20.58 \%$ ) and G1 (0 to 3 years old-9.18\%). The symptoms most frequently reported were pain (57.29\%), swelling (4.8\%), bleeding (1.97\%), infection (1.72\%) and others (1.80\%).

Trauma was responsible for 199 (17.06\%) of the recorded urgency visits. It occurred more frequently in children from G1 (34.42\% of the problems presented in this age group), and more often in males (57.62\%) than in females (42.38\%). There was a second trauma peak between 7 and 12 years of age (G3 $18.12 \%$ of the problems presented in this age group) (Figure 1). Central and lateral incisors in both permanent and primary dentitions were the most prevalent tooth types to undergo traumatic events. Maxillary central incisors were most frequently traumatized in both the primary (85.96\%) and permanent (80.60\%) dentitions.

Coronal fracture and luxation represented the most frequently observed trauma diagnosis (56.34\% and 23.68\%, respectively) (Figure 2). The most frequently teeth implicated in coronal fracture were left and right permanent maxillary central incisors (38.28\% and 36.72\%, respectively), while luxation occurred similarly both in right and left permanent (24.66\% and $20.55 \%$, respectively) and primary maxillary central incisors (17.81\% and $19.18 \%$, respectively). The main treatments performed were temporary restoration (33.33\%) for coronal fracture, and orientation (24.44\%) for luxation (Figure 3). Besides avulsion, intrusion, extrusion, bone resorption and root fracture accounted for $8.95 \%, 5.26 \%, 3.33 \%$, 3.33\% and $2.63 \%$ of the trauma diagnosis, respectively (Figure 2).

Nontraumatic events were responsible for 967 (82.92\%) of the total urgency diagnosis. The most commonly nontraumatic diagnosis was dental caries lesions (61.75\%), followed by

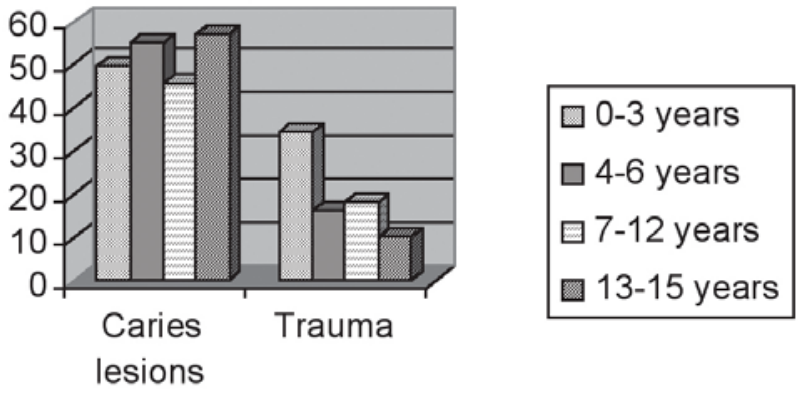

FIGURE 1- Dental caries lesions and trauma percentage in relation to total of complaints in different age groups

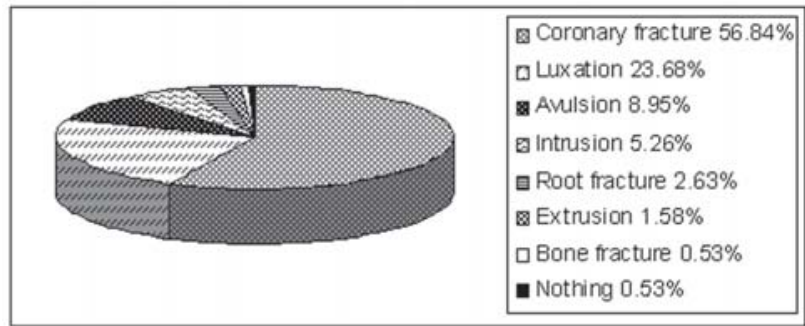

FIGURE 2- Occurrence of different types of traumatic lesions problems of eruption or root resorption (14.27\%), and bone or soft tissue lesions (6.51\%), among others (17.47\%). Significantly more children presented with carious lesions without any signs of infection, corresponding to 490 children (82.35\%), while 105 presented abscess (17.65\%).

Dental caries lesions occurred more frequently in children of G4 (57.14\%) (Figure 1). In the primary dentition, the primary mandibular second molars were the most frequent teeth affected by caries lesions (41.22\%), compared to the mandibular first molars in the permanent dentition (42.15\%). Nearly 39.39\% of

\section{Coronal fracture}

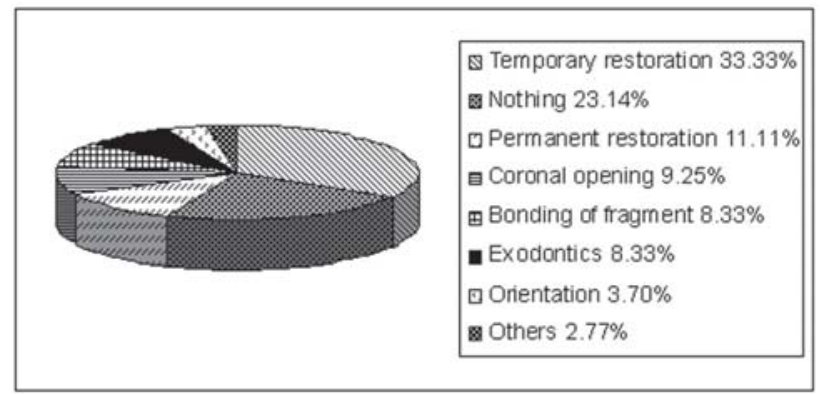

Luxation

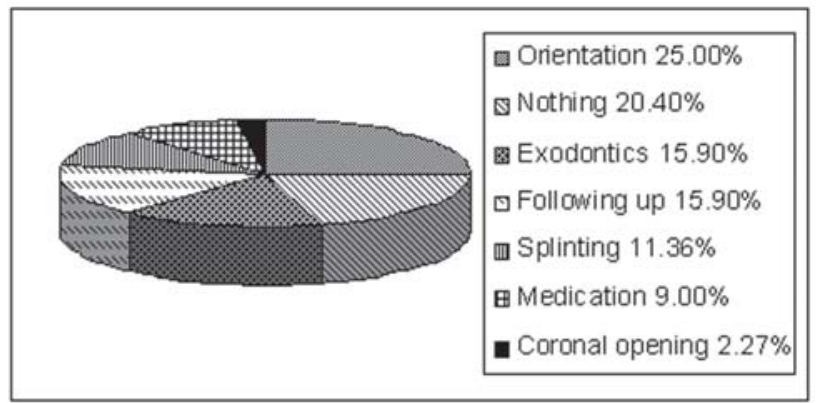

Dental caries lesions without abscess

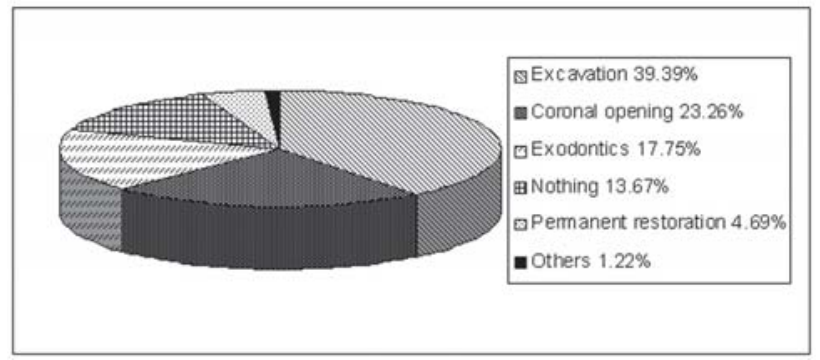

Dental caries lesions with abscess

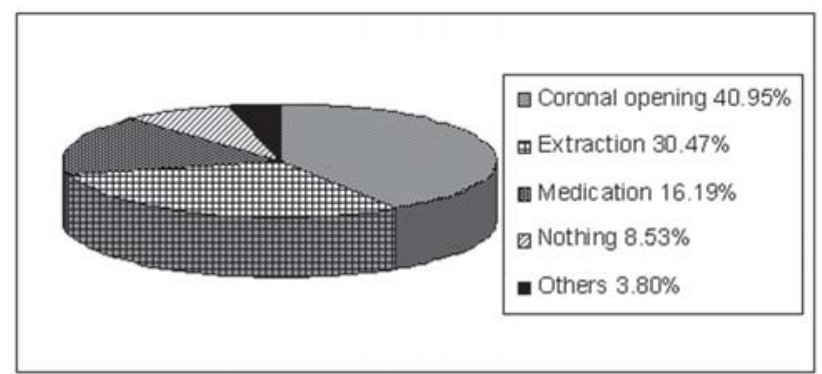

FIGURE 3- Prevalence of different treatments performed for coronal fracture, luxation, dental caries lesions without abscess and dental caries lesions with abscess 
all treatments carried out for caries lesions without abscess involved excavation and temporary restoration, and $40.95 \%$ of treatments for caries lesions with abscess were coronal opening and dressing (Figure 3).

The exams more frequently performed for all urgency appointments were clinical (99.23\%) and radiographic exams (53.60\%), since they were not mutually exclusive. However, regarding the duration of the problem presented by the patients, it was not possible to be assessed because this information was rarely recorded on their dental recordings.

Follow-up care was provided for $35.51 \%$ of all patients attending the UDS in the following clinics of the school: Pediatric Dentistry (39.62\%), Operative Dentistry (17.15\%), Urgency (13.77\%), Endodontics (10.61\%) and others (18.85\%).

\section{DISCUSSION}

The findings of this survey matches the available literature, in regard to the higher incidence of traumatic injuries affecting the primary dentition during the first three years of life $\mathrm{e}^{2,45,7,12,18}$. This significant trend may occur frequently because it is during this period of growth and development that the child is gradually moving from a stage of total dependence with respect to movement to one of independence and stability as he or she learns to sit up, bend over, crawl, kneel, stand, walk, and run. Any or all of these stages of motor development brings with it the hazard of accidental injury ${ }^{2,4,5,12}$.

The second trauma peak between 7 and 12 years of age (G3) corroborates others studies ${ }^{2,14,15}$. Frequently children at this age present increased overjet, which may increase the risk of traumatic injury. In addition, organized sports may also contribute to increased trauma episodes. Previous studies have shown that eight- to ten-year-old children are at great risk for tooth fractures and that the maxillary incisor is the most frequently fractured tooth ${ }^{2,14}$. This study yielded similar results.

Boys experienced traumatic injuries more frequently than girls. It is in accordance with some studies ${ }^{4,14,18}$. It is believed that boys between 7 to 12 years of age tend to be more active and more often involved in physical contact sports than girls. However, some other previous studies showed some different results. Glendor et al. ${ }^{10}$, in 1998, observed that there were no gender differences in type of injury and number of visits for injuries to primary and permanent teeth, while Agostini, Flaitz and Hicks ${ }^{2}$, in 2001, showed that more girls suffered traumatic dental injuries than boys.

This study has shown that maxillary teeth are more frequently traumatized than mandibular teeth. The most frequently affected teeth were the maxillary central incisors in both primary and permanent dentition traumatic injuries; this is generally supported in the existing literature $e^{4,7,9,14}$. This probably relates to vulnerable position of the maxillary central incisors. In addition, these teeth are frequently protruded and may have inadequate lip coverage.

The most prevalent type of trauma in permanent dentition was coronal fracture $\mathrm{r}^{4,10,15}$, due to the alveolar bone structural features in this dentition, which is not so resilient as in the primary ${ }^{7}$. Then, the impact is not absorbed by the bone, causing dental fracture. Nevertheless, luxation was similarly prevalent in both dentitions. Several authors reported a greater prevalence of luxation in primary teeth because of the features of bone structure ${ }^{4,7,10,15}$. However, when the mildest levels of luxation occur, many patients do not seek care, since primary dentition is not considered important to their parents, and generally there is no esthetic involvement.

In North America the urgency treatment is most frequently provided for trauma-related injuries, and caries lesions-related problems are the second most frequent complaint ${ }^{9}$. Meanwhile, our study showed different results, due to the fact that caries lesion was the most common diagnosis. It is discouraging to note that early childhood caries lesion is still a major problem for young children seeking urgency care ${ }^{2}$.

Dental caries lesion was significantly prevalent in all age groups. However, a great prevalence of caries lesions was found in G2 than G1, and in G4 than G3 due to the greatest number of primary or permanent teeth inside the mouth submitted to cariogenic challenges for a longer period of time in G2 and G4, respectively. A decrease of caries lesions prevalence from G2 to G3 was observed, which is associated to the dentition exchange, i.e., exfoliation of the decayed primary teeth and eruption of the sound teeth. In this study, an increasing trend in caries lesions prevalence according to the increase in age was observed ${ }^{5}$. The most affected teeth were molars in both dentitions due to their anatomic features and position in the mouth, which collaborate for plaque accumulation ${ }^{17}$.

The treatments proposed for traumatic and nontraumatic problems were in accordance with that indicated for this type of service, eliminating pain and avoiding complications through caries lesions excavation, temporary restorations for coronal fracture, coronal opening for periapical abscess, among others $^{17}$. As seen in Figure 3, there were situations in which no treatment was performed. Regarding caries lesions, this happened due to the complexity of such cases; these patients were referred to a specialized unit in the University. Furthermore, concerning traumatic lesions, the late seek for dental care was another reason which explains the lack of treatment.

Toothache was the most frequent complication and chief complaint of patients that seek urgency treatment both for traumatic and nontraumatic causes. Despite the efforts to improve the quality of oral health, a great part of population, mainly the one that does not have dental assistance assured, seeks dental treatment for pain and discomfort relief. So that, in most local health systems, the urgency services are the only existent and effective ${ }^{1}$.

Although pain was the major symptom reported by patients, in $42.62 \%$ of the urgency visits in the current study the children were not in pain, or their symptoms were non-specific. Almost $15 \%$ of the children presented with no chief complaint or with chief complaints that were due to physiologic events, such as permanent tooth eruption and primary tooth exfoliation. There were also some patients just seeking care for an oral evaluation or to extract some teeth due to orthodontic reasons. This preference for dental urgency appointments for non-urgency situations may reflect the "convenience" of using such appointments as a primary care source instead of regularly scheduled dental care ${ }^{2,11,18}$. Furthermore, Cangussu, et al. ${ }^{6}$, in 
2001, affirmed that the urgency treatment just means a compensatory element for the high demand of patients, but it does not contribute significantly for the improvement of the quality of oral health care and for a strong epidemiological impact on the control of oral disease in childhood. Agostini, Flaitz and Hicks², in 2001, observed that this trend to look for urgency dental appointments instead of regularly scheduled dental care also occurs in the hospital setting. These facts indicate that the dental practitioner needs to develop a screening method for determining true dental emergencies, triaging urgency care, and discerning which children really need urgent attention.

The duration of the problem presented by the patients was an important item that was not assessed because this information was not recorded in almost $95.6 \%$ of the dental recordings. It showed that sometimes the careful charting of dental recordings was neglected by the dentists working at the UDS in that period. Meanwhile, the importance of developing diagnosis by appropriate history taking and clinical examination should be emphasized by dental curricula on pediatric urgency ${ }^{17}$. Saroglu and Sonmez ${ }^{14}$, in 2002, observed that almost a half of their patients presented to their clinic after 10 days from injury time. The direct implication of this finding is that immediate treatment is a determining factor for the prognosis of the pulp and for the type of treatment to be performed. They concluded that patients who have suffered trauma with no acute symptoms tended to remain untreated as well as parents were not aware of the importance of the immediate treatment.

There are a number of barriers for seeking dental care. Dorfman, Kastner and $\mathrm{Vinci}^{8}$, in 2001, reported that access to dental care may be improved in several ways. Lack of dental care disproportionately affects poor people and those in minority groups. Opportunities should be expanded to target preventive procedures to poor inner-city and rural children through school-based programs.

\section{CONCLUSIONS}

The following conclusions can be drawn from the data presented in this paper:

- Dental caries lesion was the most prevalent problem in the studied population, followed by trauma.

- The treatments proposed for both problems were in accordance to the indicated protocol for this type of service.

- There was a large amount of non-urgent problems that were treated at UDS, such as extraction for orthodontic reasons and primary teeth exfoliation.

- Charting of most of UDS dental recordings was deficient, since there is a lack of important data such as duration of the problem presented by the patient.

- Orientation of the dental professionals working at UDS is suggested, which will enhance future evaluations for the services.

\section{AKNOWLEDGEMENTS}

The authors would like to thank Dr. Paloma Dias da Silva Telles for English review.

\section{REFERENCES}

1- Abbud R, Ferreira LA, Campos AG, Zanin KEG. Atendimento clínico de emergência: um estudo dos serviços oferecidos em dez anos. Rev Assoc Paul Cir Dent. 2002;56(4):271-5.

2- Agostini FG, Flaitz CM, Hicks MJ. Dental emergencies in a universitybased pediatric dentistry postgraduate outpatient clinic: a retrospective study. ASDC J Dent Child. 2001;68(5-6):316-21.

3- Andreasen JO. Traumatic dental injuries in children: the paradoxes of dental traumatology. Int J Paed Dent. 2000;10:181.

4- Andreasen JO. Traumatic injuries of teeth. 2a ed, Munksgaard, Copenhagen; 1981, 462p.

5- Battenhouse MAR, Nazif MM, Zullo T. Emergency care in pediatric dentistry. ASDC J Dent Child. 1988;55(1):68-71.

6- Cangussu MCT, Cabral MBBS, Liesenfeld MH, Pastor IMO. Perfil da demanda ambulatorial infantil da Faculdade de Odontologia da UFBA nos anos de 1994 e 1999. Rev FOB. 2001;9(3-4):151-5.

7- Capelozza ALA, Veltrini VC, Freitas CVJ. Prevalência de traumas em dentes anteriores num serviço de urgência odontológica. Salusvita 1999;18(1):17-26.

8- Dorfman DH, Kastner B, Vinci RJ. Dental concerns unrelated to trauma in the pediatric emergency department. Arch Pediatr Adolesc Med. 2001;155(6):699-703.

9- Fleming P, Gregg TA, Saunders IDF. Analysis of an emergency dental service provided at a children's hospital. Int J Paediatr Dent. 1991;42(2):25-30.

10- Glendor U, Halling A, Andersson L, Andreasen JO, Klitz I. Type of treatment and estimation of time spent on dental trauma: a longitudinal and retrospective study. Swed Dent J. 1998;22:47-60.

11- Graham DB, Webb MD, Seale NS. Pediatric emergency room visits for nontraumatic dental disease. Pediatr Dent. 2000;22(2):134-40.

12- Levine N. Injury to the primary dentition. Dent Clin North Am. 1982;26(3):461-80.

13- Mani SP, Cleaton-Jones PE, Lownie JF. Demographic profile of patients who present for emergency treatment at Wits' Dental School. J Dent Assoc S Afr. 1997;52(2):69-72.

14- Saroglu I, Sonmez H. The prevalence of traumatic injuries treated in the pedodontic clinic of Ankara University, Turkey, during 18 months. Dent Traumatol. 2002;18(6):299-303.

15- Schwartz S. A one-year statistical analysis of dental emergencies in a pediatric hospital. J Can Dent Assoc. 1994;60(11):959-62,966-8.

16- Scully C. The pattern of patient attendance for emergency care in British dental teaching hospital. Community Dent Health. 1995;12(3):151-4.

17- Sheller B, Williams BJ, Lombardi SM. Diagnosis and treatment of dental caries-related emergencies in children's hospital. Pediatr Dent 1997;19(8):470-5.

18- Zeng Y, Sheller B, Milgrom P. Epidemiology of dental emergency visits to an urban children’s hospital. Pediatr Dent. 1994;16(6):419-23. 